\title{
ON BAIRE-ONE MAPPINGS WITH ZERO-DIMENSIONAL DOMAINS
}

\author{
OLENA KARLOVA
}

\begin{abstract}
We generalize the Lebesgue-Hausdorff Theorem on Baire classification of mappings defined on strongly zero-dimensional spaces.
\end{abstract}

\section{INTRODUCTION}

A subset $A$ of a topological space $X$ is functionally $F_{\sigma^{-}}\left(G_{\delta}\right)$-set if $A$ is a union (an intersection) of a sequence of zero (cozero) subsets of $X$. If a set is functionally $F_{\sigma}$ and functionally $G_{\delta}$ simultaneously, then it is called functionally ambiguous.

Let $X$ and $Y$ be topological spaces and $f: X \rightarrow Y$ be a mapping. We say that $f$ belongs to

- the first Baire class, $f \in \mathrm{B}_{1}(X, Y)$, if $f$ is a pointwise limit of a sequence of continuous mappings between $X$ and $Y$;

- the first (functional) Lebesgue class, $f \in \mathrm{H}_{1}(X, Y)\left(f \in \mathrm{K}_{1}(X, Y)\right)$, if $f^{-1}(V)$ is (functionally) $F_{\sigma}$-set in $X$ for any open subset $V$ of $Y$.

Obviously, $\mathrm{H}_{1}(X, Y)=\mathrm{K}_{1}(X, Y)$ for a perfectly normal space $X$ and a topological space $Y$. It is not hard to verify that the inclusion $\mathrm{B}_{1}(X, Y) \subseteq$ $\mathrm{H}_{1}(X, Y)$ holds for any topological space $X$ and a perfectly normal space $Y$ (see [20, p. 386]). But the proof of the inverse inclusion is much more difficult problem that begins in the $\mathrm{PhD}$ thesis of René Baire [1].

The classical Lebesgue-Hausdorff theorem [22, 12] tells that

$$
\mathrm{B}_{1}(X, Y)=\mathrm{H}_{1}(X, Y)
$$

if $X$ is a metric space and $Y=[0,1]^{\omega}$, or if $X$ is a zero-dimensional metrizable separable space and $Y$ is a metrizable separable space (see [19, Theorem 24.10]). This result was generalized by many mathematicians in several ways. The first direction concerns the verification of (1.1) for a connectedlike space $Y$. So, the equality (1.1) holds under the following assumptions:

(I) $X$ is a metrizable space, $Y$ is a separable convex subset of a Banach space (S. Rolewicz [25]);

2010 Mathematics Subject Classification. Primary 26A21, 54C50; Secondary 54H05.

Key words and phrases. Baire-one mapping, $\sigma$-discrete mapping, strongly zerodimensional space. 
(II) $X$ is normal, $Y=\mathbb{R}$ (M. Laczkovich [21] without proof);

(III) $X$ is a complete metric space, $Y$ is a Banach space (C. Stegall [27]). Moreover, it was proved that if

(IV) $X$ is a topological space and $Y$ is a metrizable separable arcwise connected and locally arcwise connected space (O. Karlova, V. Mykhaylyuk [18]),

then

$$
\mathrm{B}_{1}(X, Y)=\mathrm{K}_{1}(X, Y) .
$$

R. Hansell in [8] (see also [9]) introduced the notion of $\sigma$-discrete mapping as a convenient tool for the investigation of Borel measurable mappings with valued in non-separable metric spaces. A mapping $f: X \rightarrow Y$ is called $\sigma$ discrete if there exists a family $\mathcal{B}=\bigcup_{n=1}^{\infty} \mathcal{B}_{n}$ of subsets of a space $X$ such that every family $\mathcal{B}_{n}$ is discrete in $X$ and the preimage $f^{-1}(V)$ of any open set $V$ in $Y$ is a union of sets from $\mathcal{B}$. The class of all $\sigma$-discrete mappings between $X$ and $Y$ is denoted by $\Sigma(X, Y)$. It is easy to see that if $Y$ is metrizable separable then every mapping $f: X \rightarrow Y$ is $\sigma$-discrete. The equality

$$
\mathrm{B}_{1}(X, Y)=\mathrm{H}_{1}(X, Y) \cap \Sigma(X, Y)
$$

holds in the following situations:

(V) $X$ is metrizable, $Y$ is a convex subset of a normed space (R. Hansell [9]);

(VI) $X$ is collectionwise normal, $Y$ is a closed convex subset of a Banach space (R. Hansell [11]);

(VII) $X$ is a metrizable space, $Y$ is a metrizable space, every continuous function from a closed subset of $X$ to $Y$ can be extended continuously on $X$, and for each $y \in Y$ and each neighborhood $V$ of $y$ in $Y$ there exists a neighborhood $W$ of $y$ such that each continuous function from a closed subset $F \subseteq X$ to $V$ admits an extension $f: X \rightarrow V$ (C.A. Rogers [24]);

(VIII) $X$ is a perfectly normal paracompact space, $Y$ is a Banach space (J.E. Jayne, J. Orihuela, A.J. Pallarés, G. Vera [13]);

(IX) $X$ is metrizable, $Y$ is metrizable arcwise connected and locally arcwise connected (M. Fosgerau [6]).

L. Veselý in [29] noticed that every Baire-one mapping $f$ between a topological space $X$ and a metrizable space $Y$ is "strongly $\sigma$-discrete", i.e., there exists a family $\mathcal{B}=\bigcup_{n=1}^{\infty} \mathcal{B}_{n}$ of subsets of $X$ such that for every family $\mathcal{B}_{n}=\left(B_{i}: i \in I_{n}\right)$ there exists a discrete family $\left(U_{i}: i \in I_{n}\right)$ of open sets 
in $X$ with $\overline{B_{i}} \subseteq U_{i}$ for all $i \in I_{n}$ and, moreover, the preimage $f^{-1}(V)$ of any open set $V$ in $Y$ is a union of sets from $\mathcal{B}$. The collection of all such mappings Veselý denoted by $\Sigma^{*}(X, Y)$ and proved the equality

$$
\mathrm{B}_{1}(X, Y)=\mathrm{H}_{1}(X, Y) \cap \Sigma^{*}(X, Y),
$$

in particular, in the case when

(X) $X$ is a normal space, $Y$ is a metrizable arcwise connected and locally arcwise connected space (L. Veselý [29]).

The second way of the development of the Lebesgue-Hausdorff theorem deals with the case when $Y$ does not satisfy any properties like connectedness, but $X$ is zero-dimensional, strongly zero-dimensional, etc. In this direction the following results were obtained: the equality (1.1) holds if

(XI) $X$ is a normal strongly zero-dimensional space, $Y$ is a zerodimensional metrizable separable space (H. Shatery, J. Zafarani [26]);

the equality (1.2) takes place when

(XII) $X$ is a strongly zero-dimensional space, $Y$ is a metrizable separable space (O. Karlova [14]);

and the equality (1.3) is valid if

(XIII) $X$ is a strongly zero-dimensional metrizable space and $Y$ is a metrizable space (O. Karlova [15]).

Finally, the third direction is connected with the case when $Y$ is nonmetrizable. Here we are able to prove the equality (1.1) if

(XIV) $X$ is a hereditarily Baire separable metrizable space, $Y$ is a strict inductive limit of a sequence of metrizable locally convex spaces (O. Karlova, V. Mykhaylyuk [17]).

However, it remains here many unsolved problems, in particular, the following.

Question 1.1. [23, Question 3.3, p. 659] Does every $\mathrm{H}_{1}$-mapping $f:[0,1] \rightarrow$ $C_{p}[0,1]$ belong to the first Baire class?

This question is equivalent to the following one.

Question 1.2. [23, Question 3.4, p. 659] Let $f:[0,1] \times[0,1] \rightarrow \mathbb{R}$ be a function which is continuous with respect to the first variable and belongs to the first Baire class with respect to the second one. Is $f$ a pointwise limit of a sequence of separately continuous functions? 
In this paper we develop technics from [6] and generalize the LebesgueHausdorff Theorem for $\sigma$-discrete mappings defined on strongly zero-dimensional spaces with valued in metrizable spaces. In order to do this we consider the class of $\sigma$-strongly functionally discrete mappings introduced in [16. We denote this class by $\Sigma^{f}(X, Y)$ and notice that $\Sigma^{f}(X, Y)=\Sigma^{*}(X, Y)$ if $X$ is a normal space. We prove that $\mathrm{K}_{1}(X, Y) \cap \Sigma^{f}(X, Y)=\mathrm{B}_{1}(X, Y)$, if $X$ is a strongly zero-dimensional space and $Y$ is a metrizable space. We also introduce almost strongly zero-dimensional spaces and prove that if $X$ is a topological space and $Y$ is a disconnected metrizable separable space, then the following conditions are equivalent: (i) $X$ is almost strongly zerodimensional; (ii) $\mathrm{K}_{1}(X, Y)=\mathrm{B}_{1}(X, Y)$.

2. Relations Between functionally $\sigma$-DisCREte And B B $^{\text {-MAPPings }}$

Definition 2.1. A family $\mathcal{A}=\left(A_{i}: i \in I\right)$ of subsets of a topological space $X$ is said to be

(1) discrete if every point of $X$ has a neighborhood which intersects at most one set from the family $\mathcal{A}$;

(2) strongly discrete if there exists a discrete family $\left(U_{i}: i \in I\right)$ of open subsets of $X$ such that $\overline{A_{i}} \subseteq U_{i}$ for every $i \in I$;

(3) strongly functionally discrete or, briefly, sfd-family if there exists a discrete family $\left(U_{i}: i \in I\right)$ of cozero subsets of $X$ such that $\overline{A_{i}} \subseteq U_{i}$ for every $i \in I$.

\section{Remark 2.2.}

(1) For an arbitrary space $X$ we have $(3) \Rightarrow(2) \Rightarrow(1)$;

(2) $X$ is collectonwise normal if and only if $(1)=(2)$;

(3) if $X$ is normal, then $(2)=(3)$.

Definition 2.3. Let $\mathcal{P}$ be a property of a family of sets. A family $\mathcal{A}$ is called a $\sigma-\mathcal{P}$ family if $\mathcal{A}=\bigcup_{n=1}^{\infty} \mathcal{A}_{n}$, where every family $\mathcal{A}_{n}$ has the property $\mathcal{P}$.

Definition 2.4. A family $\mathcal{B}$ of sets of a topological space $X$ is called a base for a mapping $f: X \rightarrow Y$ if the preimage $f^{-1}(V)$ of an arbitrary open set $V$ in $Y$ is a union of sets from $\mathcal{B}$.

Clearly, we may assume that $V$ is an element of an open base of $Y$ in Definition 2.4.

Definition 2.5. If a mapping $f: X \rightarrow Y$ has a base which is a $\sigma$-P family, then we say that $f$ is $a \sigma-\mathcal{P}$ mapping. 
The collection of all $\sigma$-P mappings between $X$ and $Y$ we will denote by

- $\Sigma(X, Y)$ if $\mathcal{P}$ is a property of discreteness;

- $\Sigma^{*}(X, Y)$ if $\mathcal{P}$ is a property of a strong discreteness;

- $\Sigma^{f}(X, Y)$ if $\mathcal{P}$ is a property of a strong functional discreteness;

- $\Sigma_{0}^{f}(X, Y)$ if $f$ has a $\sigma$-sfd base of zero sets.

Let us observe that a continuous mapping $f: X \rightarrow Y$ is $\sigma$-discrete if either $X$ or $Y$ is a metrizable space, since every metrizable space has a $\sigma$ discrete base of open sets. Moreover, it is evident that every mapping with values in a second countable space is $\sigma$-discrete. In [8] Hansell proved that every Borel measurable mapping $f: X \rightarrow Y$ between a complete metric space $X$ and a metric space $Y$ is $\sigma$-discrete. For any metric spaces $X$ and $Y$ the family $\Sigma(X, Y)$ is closed under pointwise limits [10], which implies that every Baire measurable mapping between metric spaces is $\sigma$-discrete.

The following fact implies from [16, Theorem 6].

Theorem 2.6. Let $X$ be a topological space and $Y$ be a metrizable space. Then $\mathrm{K}_{1}(X, Y) \cap \Sigma^{f}(X, Y)=\Sigma_{0}^{f}(X, Y)$.

The next simple lemma will be useful.

Lemma 2.7. Let $X$ be a topological space, $\left(U_{i}: i \in I\right)$ be a locally finite family of cozero subsets of $X,\left(F_{i}: i \in I\right)$ be a family of zero subsets of $X$ such that $F_{i} \subseteq U_{i}$ for every $i \in I$. Then $F=\bigcup_{i \in I} F_{i}$ is a zero set in $X$.

Proof. For every $i \in I$ we choose a continuous function $f_{i}: X \rightarrow[0,1]$ such that $F_{i}=f_{i}^{-1}(0)$ and $X \backslash U_{i}=f_{i}^{-1}(1)$. For every $x \in X$ let $f(x)=\min _{i \in I} f_{i}(x)$. Then $f: X \rightarrow[0,1]$ is continuous and $F=f^{-1}(0)$.

Corollary 2.8. A union of an sfd-family of zero sets in a topological space is a zero set.

We say that a topological space $X$ is strongly zero-dimensional if for any completely separated subsets $A$ and $B$ of $X$ there exists a clopen set $U$ such that $A \subseteq U \subseteq X \backslash B$.

For families $\mathcal{A}$ and $\mathcal{B}$ we write $\mathcal{A} \prec \mathcal{B}$ if for every $A \in \mathcal{A}$ there exists $B \in \mathcal{B}$ such that $A \subseteq B$.

Proposition 2.9. Let $X$ be a strongly zero-dimensional space, $(Y, d)$ be a metric space, $f: X \rightarrow Y$ be a mapping, $\mathcal{F}_{1}, \ldots, \mathcal{F}_{n}$ be families of zero subsets of $X$ such that

(1) $\mathcal{F}_{k}$ is an sfd-family for every $k=1, \ldots, n$; 
(2) $\mathcal{F}_{k+1} \prec \mathcal{F}_{k}$ for every $k=1, \ldots, n-1$;

(3) for every $k=1, \ldots, n$ the inequality $\operatorname{diam}(f(F))<\frac{1}{2^{k+2}}$ holds for all $F \in \mathcal{F}_{k}$.

Then there exists a continuous mapping $g: X \rightarrow Y$ such that the inclusion $x \in \cup \mathcal{F}_{k}$ for some $k=1, \ldots, n$ implies the inequality

$$
d(f(x), g(x))<\frac{1}{2^{k}} .
$$

Proof. Let $\mathcal{F}_{k}=\left(F_{i, k}: i \in I_{k}\right), k=1, \ldots, n$. We choose a discrete family $\left(U_{i, 1}: i \in I_{1}\right)$ of cozero sets in $X$ such that $F_{i, 1} \subseteq U_{i, 1}$ for every $i \in I_{1}$. Let $\left(V_{i, 1}: i \in I_{1}\right)$ be a family of clopen sets such that $F_{i, 1} \subseteq V_{i, 1} \subseteq U_{i, 1}$. Now we take a discrete family $\left(G_{i, 2}: i \in I_{2}\right)$ of cozero sets such that $F_{i, 2} \subseteq G_{i, 2}$ for every $i \in I_{2}$. Since $\mathcal{F}_{2} \prec \mathcal{F}_{1}$, for every $i \in I_{2}$ there exists unique $j \in I_{1}$ such that $F_{i, 2} \subseteq F_{j, 1}$. We denote $U_{i, 2}=G_{i, 2} \cap V_{j, 1}$ and choose a clopen set $V_{i, 2}$ with $F_{i, 2} \subseteq V_{i, 2} \subseteq U_{i, 2} \subseteq V_{j, 1}$. Proceeding in this way we obtain discrete families $\left(U_{i, k}: i \in I_{k}\right)$ and $\left(V_{i, k}: i \in I_{k}\right)$ of subsets of $X$ for $k=1, \ldots, n$ such that $U_{i, k}$ is a cozero set, $V_{i, k}$ is a clopen set, for every $k=1, \ldots, n-1$ and $i \in I_{k+1}$ there exists unique $j \in I_{k}$ such that

$$
\begin{gathered}
F_{i, k+1} \subseteq F_{j, k}, \\
F_{i, k+1} \subseteq V_{i, k+1} \subseteq U_{i, k+1} \subseteq V_{j, k} .
\end{gathered}
$$

Observe that for every $k$ the set $V_{k}=\bigcup_{i \in I_{k}} V_{i, k}$ is clopen by Corollary 2.8,

Let $y_{0} \in f(X)$ and $y_{i, k} \in f\left(F_{i, k}\right)$ be arbitrary points for every $k$ and $i \in I_{k}$. For all $x \in X$ let

$$
g_{0}(x)=y_{0} .
$$

Suppose that for some $k, 1 \leq k<n$, we have defined continuous mappings $g_{1}, \ldots, g_{k}$ such that

$$
g_{k}(x)= \begin{cases}g_{k-1}(x), & \text { if } x \in X \backslash V_{k}, \\ y_{i, k}, & \text { if } x \in V_{i, k} \text { for some } i \in I_{k} .\end{cases}
$$

Let

$$
g_{k+1}(x)= \begin{cases}g_{k}(x), & \text { if } x \in X \backslash V_{k+1}, \\ y_{i, k+1}, & \text { if } x \in V_{i, k+1} \text { for some } i \in I_{k+1} .\end{cases}
$$

Then the mapping $g_{k+1}: X \rightarrow Y$ is continuous, since every restriction $\left.g_{k+1}\right|_{V_{k+1}}$ and $\left.g_{k+1}\right|_{X \backslash V_{k+1}}$ is continuous and the set $V_{k+1}$ is clopen. Proceeding inductively we define continuous mappings $g_{1}, \ldots, g_{n}$ satisfying (2.4).

We put $g=g_{n}$ and prove that $g$ satisfies (2.1). We first show that

$$
d\left(g_{k+1}(x), g_{k}(x)\right)<\frac{1}{2^{k}}
$$


for all $0 \leq k<n$ and $x \in X$. Indeed, if $x \in X \backslash V_{k+1}$, then $g_{k+1}(x)=g_{k}(x)$ and $d\left(g_{k+1}(x), g_{k}(x)\right)=0$. Assume $x \in V_{i, k+1}$ for some $i \in I_{k+1}$. Take $j \in I_{k}$ such that conditions (2.2) and (2.3) hold. Then $g_{k+1}(x)=y_{i, k+1}$ and $g_{k}(x)=$ $y_{j, k}$. Since $f\left(F_{i, k+1}\right) \subseteq f\left(F_{j, k}\right), y_{i, k+1} \in f\left(F_{j, k}\right)$. Hence, $d\left(g_{k+1}(x), g_{k}(x)\right) \leq$ $\operatorname{diam}\left(f\left(F_{j, k}\right)\right)<\frac{1}{2^{k+2}}$.

Let $1 \leq k \leq n$ and $x \in \cup \mathcal{F}_{k}$. Then $x \in F_{i, k} \subseteq V_{i, k}$ for some $i \in I_{k}$. It follows that $g_{k}(x)=y_{i, k}$ and $d\left(f(x), g_{k}(x)\right) \leq \operatorname{diam}\left(f\left(F_{i, k}\right)\right)<\frac{1}{2^{k+2}}$. Taking into account (2.5), we obtain that

$$
d(f(x), g(x)) \leq d\left(f(x), g_{k}(x)\right)+\sum_{i=k}^{n-1} d\left(g_{i}(x), g_{i+1}(x)\right)<\frac{1}{2^{k+2}}+\frac{1}{2^{k+1}}<\frac{1}{2^{k}}
$$

Theorem 2.10. Let $X$ be a strongly zero-dimensional space and $Y$ be a metrizable space. Then $\Sigma_{0}^{f}(X, Y) \subseteq \mathrm{B}_{1}(X, Y)$.

Proof. Fix a metric $d$ on $Y$ which generates its topological structure. For every $k \in \mathbb{N}$ we consider a covering $\mathcal{U}_{k}$ of $Y$ by open sets with diameters at most $\frac{1}{2^{k}}$. Let $f \in \Sigma_{0}^{f}(X, Y)$ and $\mathcal{B}$ be a $\sigma$-sfd base for $f$, which consists of zero subsets of $X$. For every $k \in \mathbb{N}$ we put

$$
\mathcal{B}_{k}=\left(B \in \mathcal{B}: \exists U \in \mathcal{U}_{k} \mid B \subseteq f^{-1}(U)\right) .
$$

Then $\mathcal{B}_{k}$ is a $\sigma$-sfd family and $X=\cup \mathcal{B}_{k}$ for every $k$. According to $[16$, Lemma 13] for every $k \in \mathbb{N}$ there exists a sequence $\left(\mathcal{B}_{k, n}\right)_{n=1}^{\infty}$ of sfd families of zero subsets of $X$ such that $\mathcal{B}_{k, n} \prec B_{k}, \mathcal{B}_{k, n} \prec \mathcal{B}_{k, n+1}$ for every $n \in \mathbb{N}$ and $\bigcup \bigcup_{n=1}^{\infty} \mathcal{B}_{k, n}=X$. For all $k, n \in \mathbb{N}$ we set

$$
\mathcal{F}_{k, n}=\left(B_{1} \cap \cdots \cap B_{k}: B_{m} \in \mathcal{B}_{m, n}, 1 \leq m \leq k\right) .
$$

Notice that each of the families $\mathcal{F}_{k, n}$ is strongly functionally discrete, consists of zero sets and satisfy the following conditions:

(a) $\mathcal{F}_{k+1, n} \prec \mathcal{F}_{k, n}$,

(b) $\mathcal{F}_{k, n} \prec \mathcal{F}_{k, n+1}$,

(c) $\bigcup_{n=1}^{\infty} \mathcal{F}_{k, n}=X$.

For every $n \in \mathbb{N}$ we apply Proposition 2.9 to $f$ and to the families $\mathcal{F}_{1, n}$, $\mathcal{F}_{2, n}, \ldots, \mathcal{F}_{n, n}$. We get a sequence of continuous mappings $g_{n}: X \rightarrow Y$ such that the inclusion $x \in \cup \mathcal{F}_{k, n}$ for some $k \leq n$ implies $d\left(f(x), g_{n}(x)\right)<\frac{1}{2^{k}}$. It is easy to see that properties (b) and (c) imply that $g_{n} \rightarrow f$ pointwise on $X$. Hence, $f \in \mathrm{B}_{1}(X, Y)$.

Proposition 2.11. Let $X$ be a topological space and $Y$ be a metrizable space. Then $\mathrm{B}_{1}(X, Y) \subseteq \Sigma_{0}^{f}(X, Y)$. 
Proof. Let $f \in \mathrm{B}_{1}(X, Y)$ and $\left(f_{n}\right)_{n=1}^{\infty}$ be a sequence of continuous mappings $f_{n}: X \rightarrow Y$ such that $f(x)=\lim _{n \rightarrow \infty} f_{n}(x)$ for all $x \in X$. Let $\mathcal{V}=\bigcup_{m=1}^{\infty} \mathcal{V}_{m}$ be a $\sigma$-discrete open base of the space $Y$. For every $V \in \mathcal{V}$ we choose a sequence $\left(G_{k, V}\right)_{k=1}^{\infty}$ of open sets such that $\overline{G_{k, V}} \subseteq G_{k+1, V}$ for every $k \in \mathbb{N}$ and $V=\bigcup_{k=1}^{\infty} \overline{G_{k, V}}$. It is not hard to verify that

$$
f^{-1}(V)=\bigcup_{k=1}^{\infty} \bigcap_{n=k}^{\infty} f_{n}^{-1}\left(\overline{G_{k, V}}\right)
$$

Denote $F_{k, V}=\bigcap_{n=k}^{\infty} f_{n}^{-1}\left(\overline{G_{k, V}}\right)$ and notice that every $F_{k, V}$ is a zero set in $X$. For all $k, m \in \mathbb{N}$ we put $\mathcal{B}_{k, m}=\left(F_{k, V}: V \in \mathcal{V}_{m}\right)$ and $\mathcal{B}=\bigcup_{k, m=1}^{\infty} \mathcal{B}_{k, m}$. Then $\mathcal{B}$ is a base for $f$. Moreover, every family $\mathcal{B}_{k, m}$ is strongly functionally discrete, since $F_{k, V} \subseteq f_{k}^{-1}(V)$ and the family $\left(f_{k}^{-1}(V): V \in \mathcal{V}_{m}\right)$ is discrete and consists of cozero sets.

Combining Theorems 2.6, 2.10 and Proposition 2.11, we get

Theorem 2.12. Let $X$ be a strongly zero-dimensional space and $Y$ be a metrizable space. Then $\mathrm{K}_{1}(X, Y) \cap \Sigma^{f}(X, Y)=\mathrm{B}_{1}(X, Y)$.

According to Theorem 3 from [8] we have $\mathrm{K}_{1}(X, Y) \subseteq \Sigma^{f}(X, Y)$ for any completely metrizable $X$ and metrizable $Y$. This fact and Theorem 2.12 immediately imply the following result.

Theorem 2.13. Let $X$ be a completely metrizable strongly zero-dimensional space and $Y$ be a metrizable space. Then $\mathrm{K}_{1}(X, Y)=\mathrm{B}_{1}(X, Y)$.

We show that the metrizability of $Y$ in Theorem 2.13 is essential.

Example 2.14. There exists a completely metrizable strongly zerodimensional space $X$ and a Lindelöf strongly zero-dimensional space $Y$ such that $\mathrm{K}_{1}(X, Y) \backslash \mathrm{B}_{1}(X, Y) \neq \emptyset$.

Proof. Let $X$ be the set of all irrational numbers with the euclidian topology and $Y$ be the same set with the topology induced from the Sorgenfrey line (recall that the Sorgenfrey line is the real line $\mathbb{R}$ endowed with the topology generated by the base consisting of all semi-intervals $[a, b)$, where $a<b$ ).

Take a countable dense in $X$ set $Q=\left\{q_{n}: n \in \mathbb{N}\right\}$ and for all $x \in X$ we put

$$
f(x)= \begin{cases}x-\frac{1}{n}, & \text { if } x=q_{n} \\ x, & \text { if } x \in X \backslash Q .\end{cases}
$$


To show that $f \in \mathrm{K}_{1}(X, Y)$ it is enough to verify that $f^{-1}([a, b) \cap Y)$ is an $F_{\sigma}$-set in $X$ for every $a<b$, since $Y$ is Lindelöf. Denote $E=f^{-1}([a, b) \cap Y)$. Notice that the sets $A=(X \cap[a, b)) \backslash E$ and $B=E \backslash(a, b)$ are subsets of $Q$. Let $\left(n_{k}\right)_{k=1}^{\infty}$ be an increasing sequence of numbers such that $A=\left\{q_{n_{k}}\right.$ : $k \in \mathbb{N}\}$. Then $a \leq r_{n_{k}} \leq a+\frac{1}{n_{k}}$ for every $k$. Therefore, $\lim _{k \rightarrow \infty} r_{n_{k}}=a$ in $X$. Hence, the set $A$ is $G_{\delta}$ in $X$. Then the equality $E=(X \cap[a, b) \backslash A) \cup B$ implies that $E$ is an $F_{\sigma}$-set in $X$.

Now we prove that $f \notin \mathrm{B}_{1}(X, Y)$. Assume that there exists a sequence of continuous mappings $f_{n}: X \rightarrow Y$ such that $f_{n}(x) \rightarrow f(x)$ for every $x \in X$. Let

$$
A_{n}=\left\{x \in X: \forall k \geq n \quad f_{k}(x) \geq f(x)\right\}
$$

for $n \in \mathbb{N}$. It is easy to see that $\bigcup_{n=1}^{\infty} A_{n}=X$. Since the set $X \backslash Q$ is of the second category in $X$, there exist a number $n$ and a set $[a, b]$ such that $[a, b] \cap X \subseteq \overline{A_{n} \backslash Q}$. Notice that $A_{n} \backslash Q \subseteq F$, where $F=\bigcap_{k=n}^{\infty}\{x \in$ $\left.X: f_{k}(x) \geq x\right\}$. Since $F$ is closed in $X$, we have $[a, b] \cap X \subseteq F$. Then $f(x)=\lim _{k \rightarrow \infty} f_{k}(x) \geq x$ for all $x \in[a, b] \cap X$, a contradiction.

\section{Almost Strongly ZERO-Dimensional SPACES AND}

\section{CHARACTERIZATION THEOREMS}

In this section we find necessary conditions on a space $X$ under which the equality $\mathrm{K}_{1}(X, Y) \cap \Sigma^{f}(X, Y)=\mathrm{B}_{1}(X, Y)$ holds for any disconnected metrizable space $Y$.

Definition 3.1. A subset $F$ of a topological space $X$ is called $a C$-set if there exists a sequence $\left(U_{n}\right)_{n=1}^{\infty}$ of clopen sets in $X$ such that $F=\bigcap_{n=1}^{\infty} U_{n}$. A set is called $a C_{\sigma}$-set if it is a union of a sequence of $C$-sets.

Definition 3.2. We say that a topological space $X$ is almost strongly zerodimensional if every zero subset of $X$ is a $C_{\sigma}$-set.

Notice that every strongly zero-dimensional space is almost strongly zerodimensional.

Lemma 3.3. Let $X$ be a topological space, $C_{1}$ and $C_{2}$ be disjoint $C$-subsets of $X$. Then there exists a clopen set $G$ in $X$ such that $C_{1} \subseteq G \subseteq X \backslash C_{2}$.

Proof. Let $\left(U_{n}\right)_{n=1}^{\infty}$ and $\left(V_{n}\right)_{n=1}^{\infty}$ be sequences of clopen subsets of $X$ such that $X \backslash C_{1}=\bigcup_{n=1}^{\infty} U_{n}$ and $X \backslash C_{2}=\bigcup_{n=1}^{\infty} V_{n}$. For every $n \in \mathbb{N}$ put $G_{n}=$ $V_{n} \backslash \bigcup_{k=1}^{n} U_{k}$ and let $G=\bigcup_{n=1}^{\infty} G_{n}$. Clearly, $C_{1} \subseteq G \subseteq X \backslash C_{2}$ and $G$ is open in 
$X$. It remains to show that $G$ is closed. Let $x \in \bar{G}$. If $x \in C_{1}$ then $x \in G$. If $x \notin C_{1}$, then there is $N \in \mathbb{N}$ such that $x \in U_{N}$. Notice that $U_{N} \cap G_{n}=\emptyset$ for all $n \geq N$. Then $x \in \overline{\bigcup_{n=1}^{N-1} G_{n}}=\bigcup_{n=1}^{N-1} \overline{G_{n}}=\bigcup_{n=1}^{N-1} G_{n} \subseteq G$.

Corollary 3.4. Let $X$ be a topological space and $C_{1}, \ldots, C_{n}$ be disjoint $C$ subsets of $X, n \in \mathbb{N}$. Then there exist disjoint clopen sets $G_{1}, \ldots, G_{n}$ in $X$ such that $X=G_{1} \cup \cdots \cup G_{n}$ and $C_{i} \subseteq G_{i}$ for every $i=1, \ldots, n$.

Proposition 3.5. Every almost strongly zero-dimensional completely regular space $X$ is totally separated.

Proof. Let $x, y \in X$ be distinct points and $U, V$ be disjoint zero neighborhoods of $x$ and $y$, respectively. Since $X$ is almost strongly zero-dimensional, there exist $C$-sets $C_{x}$ and $C_{y}$ such that $x \in C_{x} \subseteq U$ and $y \in C_{y} \subseteq V$. By Lemma 3.3 there exists a clopen set $G$ such that $C_{x} \subseteq G$ and $G \cap C_{y}=\emptyset$. Hence, $x$ and $y$ can be separated by a clopen set which implies that $X$ is totally separated.

Lemma 3.6. Let $X$ be a topological space, $F \subseteq X$ be a countably compact $C_{\sigma}$-set, $C \subseteq X$ be a $C$-set and $F \cap C=\emptyset$. Then there exists a clopen set $G$ in $X$ such that $F \subseteq G \subseteq X \backslash C$.

Proof. Let $\left(C_{n}\right)_{n=1}^{\infty}$ be an increasing sequence of $C$-sets such that $F=$ $\bigcup_{n=1}^{\infty} C_{n}$. Lemma 3.3 implies that for every $n$ there exists a clopen set $G_{n}$ in $X$ such that $C_{n} \subseteq G_{n} \subseteq X \backslash C$. Since $F$ is countably compact, we choose a finite subcovering $\mathcal{G}$ of the covering $\left(G_{n}: n \in \mathbb{N}\right)$ of $F$. It remains to put $G=\cup \mathcal{G}$.

In the same manner we can prove the following result.

Lemma 3.7. Let $X$ be a topological space and $F, E \subseteq X$ be disjoint countably compact $C_{\sigma}$-sets. Then there exists a clopen set $G$ in $X$ such that $F \subseteq G \subseteq X \backslash E$.

Taking into account that every closed subset of a countably compact space is countably compact, we obtain the following corollary from Lemma 3.7 .

Proposition 3.8. Let $X$ be a countably compact space. Then $X$ is almost strongly zero-dimensional if and only if it is strongly zero-dimensional.

The following question is open. 
Question 3.9. Do there exists a completely regular almost dimensional space which is not strongly zero-dimensional?

Proposition 3.10. Let $X$ be a topological space, $Y$ be a disconnected space such that $\mathrm{K}_{1}(X, Y) \cap \Sigma^{f}(X, Y) \subseteq \mathrm{B}_{1}(X, Y)$. Then $X$ is almost strongly zero-dimensional.

Proof. Let $U$ and $V$ be clopen disjoint nonempty subsets of $Y$ such that $Y=U \cup V, F \subseteq X$ be a zero set, $y_{1} \in U, y_{2} \in V$ and let $f: X \rightarrow Y$ be a mapping such that $f(x)=y_{1}$ for all $x \in F$ and $f(x)=y_{2}$ for all $x \in X \backslash F$. It is easy to see that $f \in \mathrm{K}_{1}(X, Y) \cap \Sigma^{f}(X, Y)$. Then there exists a sequence $\left(f_{n}\right)_{n=1}^{\infty}$ of continuous mappings $f_{n}: X \rightarrow Y$ such that $\lim _{n \rightarrow \infty} f_{n}(x)=f(x)$ for every $x \in X$. Then $F=f^{-1}(U)=\bigcup_{n=1}^{\infty} \bigcap_{m=n}^{\infty} f_{m}^{-1}(U)$. Hence, $F$ is a $C_{\sigma}$-set.

Theorem 3.11. Let $Y$ be a disconnected metrizable space. If

(a) $X$ is locally compact paracompact Hausdorff space, or

(b) $X$ is a countably compact space,

then the following conditions are equivalent:

(1) $\mathrm{K}_{1}(X, Y) \cap \Sigma^{f}(X, Y)=\mathrm{B}_{1}(X, Y)$;

(2) $X$ is a strongly zero-dimensional space.

Proof. $(1) \Rightarrow(2)$. According to Proposition 3.10, $X$ is almost strongly zerodimensional. It follows that $X$ is strongly zero-dimensional in case (b) by Proposition 3.8 . In case (a) $X$ is completely regular and, consequently, totally separated by Proposition 3.5. It remains to apply Theorem 6.2.10 from [5].

The implication $(2) \Rightarrow(1)$ follows from Theorem 2.12 .

A sequence $\left(f_{n}\right)_{n=1}^{\infty}$ of mappings $f_{n}: X \rightarrow Y$ is called stably convergent to a mapping $f: X \rightarrow Y$ if for every $x \in X$ there exists a number $n_{0}$ such that $f_{n}(x)=f(x)$ for all $n \geq n_{0}$. We denote this fact by $f_{n} \stackrel{s t}{\longrightarrow} f$.

Lemma 3.12. Let $X$ be an almost zero-dimensional space, $Y$ be a $T_{1}$-space and $f \in \mathrm{K}_{1}(X, Y)$ be a finite-valued mapping. Then there exists a sequence of continuous finite-valued mappings $f_{n}: X \rightarrow Y$ which is stably convergent to $f$ on $X$.

Proof. Denote $f(X)=\left\{y_{1}, \ldots, y_{m}\right\}$. Since for every $1 \leq i \leq m$ the set $A_{i}=$ $f^{-1}\left(y_{i}\right)$ is functionally $F_{\sigma}$ in $X$, there exists an increasing sequence $\left(C_{i, n}\right)_{n=1}^{\infty}$ of $C$-subsets of $X$ such that $A_{i}=\bigcup_{n=1}^{\infty} C_{i, n}$. According to Corollary 3.4 for 
every $n \in \mathbb{N}$ there are disjoint clopen sets $G_{1, n}, \ldots, G_{m, n}$ such that $C_{i, n} \subseteq$ $G_{i, n}$ for every $i=1, \ldots, m$ and $X=G_{1, n} \cup \cdots \cup G_{m, n}$. Now for every $n \geq 1$ we put $f_{n}(x)=y_{i}$ if $x \in G_{i, n}$ for some $i=1, \ldots, m$. It is easy to see that $f_{n} \stackrel{s t}{\longrightarrow} f$ on $X$.

Lemma 3.13. Let $X$ be an almost zero-dimensional space, $(Y, d)$ be a metric space and $\left(f_{n}\right)_{n=1}^{\infty}$ be a sequence of finite-valued mappings $f_{n} \in \mathrm{K}_{1}(X, Y)$ which is uniformly convergent to $f: X \rightarrow Y$. Then $f \in \mathrm{B}_{1}(X, Y)$.

Proof. Without loss of generality we may assume that

$$
d\left(f_{n+1}(x), f_{n}(x)\right) \leq \frac{1}{2^{n+1}}
$$

for all $x \in X$ and $n \in \mathbb{N}$. By Lemma 3.12 for every $n \in \mathbb{N}$ there exists a sequence $\left(f_{n, m}\right)_{m=1}^{\infty}$ of continuous finite-valued mappings $f_{n, m}: X \rightarrow Y$ such that

$$
f_{n, m} \stackrel{d}{\longrightarrow} f_{n}
$$

For all $x \in X$ and $m \in \mathbb{N}$ we put

$$
h_{0, m}(x)=h_{1, m}(x)=f_{1, m}(x) .
$$

Now assume that for some $k \in \mathbb{N}$ we have already defined sequences $\left(h_{1, m}\right)_{m=1}^{\infty}, \ldots,\left(h_{k, m}\right)_{m=1}^{\infty}$ of continuous finite-valued mappings such that

$$
h_{n, m} \stackrel{d}{\longrightarrow} f_{n} \text { for all } n=1, \ldots, k,
$$

$$
d\left(h_{n+1, m}(x), h_{n, m}(x)\right) \leq \frac{1}{2^{n+1}} \text { for all } x \in X, m \in \mathbb{N} \text { and } n=0, \ldots, k-1 .
$$

For every $m \in \mathbb{N}$ let

$$
U_{m}=\left\{x \in X: d\left(f_{k+1, m}(x), h_{k, m}(x)\right) \leq \frac{1}{2^{k+1}}\right\} .
$$

Then $U_{m}$ is clopen in $X$. Moreover, conditions (3.1), (3.2) and (3.3) imply that

$$
X=\bigcup_{n=1}^{\infty} \bigcup_{m=n}^{\infty} U_{m}
$$

Define a sequence of finite-valued continuous mappings $\left(h_{k+1, m}\right)_{m=1}^{\infty}$ by the formula

$$
h_{k+1, m}(x)= \begin{cases}f_{k+1, m}(x), & \text { if } x \in U_{m}, \\ h_{k, m}(x), & \text { if } x \notin U_{m} .\end{cases}
$$

Notice that (3.2) and (3.5) imply that $h_{k+1, m} \stackrel{d}{\longrightarrow} f_{k+1}$ on $X$.

We prove that the inequality (3.4) holds. Fix $m \in \mathbb{N}$ and $x \in X$. If $x \in U_{m}$, then $h_{k+1, m}(x)=f_{k+1, m}(x)$ and $d\left(h_{k+1, m}(x), h_{k, m}(x)\right)=$ 
$d\left(f_{k+1, m}(x), h_{k, m}(x)\right) \leq \frac{1}{2^{k+1}}$. If $x \notin U_{m}$, then $h_{k+1, m}(x)=h_{k, m}(x)$ i $d\left(h_{k+1, m}(x), h_{k, m}(x)\right)=0$.

Finally, we show that $\lim _{m \rightarrow \infty} h_{m, m}(x)=f(x)$. Let $x \in X, \varepsilon>0$ and $n_{0}$ be a number such that

$$
\frac{1}{2^{n_{0}}}<\frac{\varepsilon}{2} \quad \text { and } \quad d\left(f_{n_{0}}(x), f(x)\right)<\frac{\varepsilon}{2} .
$$

Take $m_{0}>n_{0}$ with $h_{n_{0}, m}(x)=f_{n_{0}}(x)$ for all $m \geq m_{0}$. Then

$$
\begin{gathered}
d\left(h_{m, m}(x), f(x)\right) \leq \\
\leq \sum_{i=n_{0}+1}^{m} d\left(h_{i-1, m}(x), h_{i, m}(x)\right)+d\left(h_{n_{0}, m}(x), f_{n_{0}}(x)\right)+d\left(f_{n_{0}}(x), f(x)\right)< \\
<\sum_{i=n_{0}+1}^{m} \frac{1}{2^{i}}+\frac{\varepsilon}{2}<\frac{1}{2^{n_{0}}}+\frac{\varepsilon}{2}<\varepsilon
\end{gathered}
$$

for all $m \geq m_{0}$. Hence, $f \in \mathrm{B}_{1}(X, Y)$.

Theorem 3.14. Let $X$ be an almost zero-dimensional space and $Y$ be a metrizable separable space. Then $\mathrm{K}_{1}(X, Y)=\mathrm{B}_{1}(X, Y)$.

Proof. The inclusion $\mathrm{B}_{1}(X, Y) \subseteq \mathrm{K}_{1}(X, Y)$ follows from the equality (2.6).

Let $f \in \mathrm{K}_{1}(X, Y)$ and $d$ be a metric on $Y$ such that $(Y, d)$ is completely bounded. For every $n \in \mathbb{N}$ we take a finite $\frac{1}{n}$-network $Y_{n}=\left\{y_{i, n}: i \in I_{n}\right\}$ in $Y$ and put $A_{i, n}=\left\{x \in X: d\left(f(x), y_{i, n}\right)<\frac{1}{n}\right\}$ for $n \in \mathbb{N} i \in I_{n}$. Notice that for every $n$ the family $\left(A_{i, n}: i \in I_{n}\right)$ is a covering of $X$ by functionally $F_{\sigma}$-sets. Similarly as in the proof of the Reduction Theorem [20, p. 350] we take a sequence of disjoint functionally ambiguous sets $F_{i, n}$ in $X$ such that $F_{i, n} \subseteq A_{i, n}$ and $\bigcup_{i \in I_{n}} F_{i, n}=X$. For every $n \in \mathbb{N}$ we put $f_{n}(x)=y_{i, n}$ if $x \in$ $F_{i, n}$ for some $i \in I_{n}$. Then $\left(f_{n}\right)_{n=1}^{\infty}$ is a sequence of finite-valued mappings $f_{n} \in \mathrm{K}_{1}(X, Y)$ which is uniformly convergent to $f$ on $X$. It remains to apply Lemma 3.12 .

Combining Proposition 3.10 and Theorem 3.14 we obtain the following result.

Theorem 3.15. If $X$ is a topological space and $Y$ is a disconnected metrizable separable space, then the following conditions are equivalent:

(1) $X$ is almost strongly zero-dimensional;

(2) $\mathrm{K}_{1}(X, Y)=\mathrm{B}_{1}(X, Y)$.

\section{REFERENCES}

[1] R. Baire, Sur les fonctions de variables reélles, Ann. Mat. Pura Appl., ser. 3. (3) (1899), 1-123. 
[2] S. Banach, Üver analytisch darstellbare Operationen in abstrakten Räumen, Fund. Math. 17 (1) (1931), 283-295.

[3] R. Blair, A. Hager, Extensions of zero-sets and of real-valued functions, Mathematische Zeitschrift 136 (1974), 41-52.

[4] H. Corson, Normality in subsets of product spaces, Amer. J. Math. 81 (1959), 785796.

[5] R. Engelking, General Topology, Revised and completed edition. Heldermann Verlag, Berlin, 1989.

[6] M. Fosgerau, When are Borel functions Baire functions?, Fund. Math. 143 (1993), $137-152$.

[7] K. Garg, On the classification of set-valued functions, Real Anal. Exch. 9 (1985), 86-93.

[8] R.W. Hansell, Borel measurable mappings for nonseparable metric spaces, Trans. Amer. Math. Soc. 161 (1971), 145-169.

[9] R.W. Hansell, On Borel mappings and Baire functions, Trans. Amer. Math. Soc. 194 (1974), 145-169.

[10] R.W. Hansell, Extended Bochner measurable selectors, Math. Ann. 277 (1987), 7994.

[11] R. Hansell, First class functions with values in nonseparable spaces, in: Constantin Carathéodory: An International Tribute, T. M. Rassias (ed.), World Sci., Singapore, (1992), 461-475.

[12] F. Hausdorff, Set Theory, American Mathematical Soc., 1957.

[13] J.E. Jayne, J. Orihuela, A.J. Pallarés, G. Vera, $\sigma$-fragmentability of multivalued maps and selection theorems, J. Fuct. Anal. 117 (2) (1993), 243-273.

[14] O. Karlova, Disconnected spaces and Baire classification of Lebesgue one mappings, Ukr. Math. Bull. 4 (2) (2007), 180-188 (in Ukrainian).

[15] O. Karlova, Baire classification of $\sigma$-discrete mappings, Bull. Chernivtsi Nat. Univ. Mathematics, 374 (2008), 52-54 (in Ukrainian).

[16] O. Karlova, Functionally $\sigma$-discrete mappings and a generalization of Banach's theorem, arXiv:1501.02901.

[17] O. Karlova, V. Mykhaylyuk, On Baire one mappings and Lebesgue one mappings with values in inductive limits, Mat. Studii 25 (1) (2006), 103-107.

[18] O. Karlova, V. Mykhaylyuk, Functions of the first Baire class with values in metrizable spaces, Ukr. J. Math. 58 (4) (2006), 567-571 (in Ukrainian).

[19] A. Kechris, Classical Descriptive Set Theory, Graduate Texts in Mathematics, 156, Springer, 1995.

[20] K. Kuratowski, Topology. Volume 1, Academic Press, 1966.

[21] M. Laczkowich, Baire 1 functions, Real Anal. Exch. (9) (1983-1984), 15-28.

[22] H. Lebesgue, Sur les fonctions représentables analytiqment, J. Math. 1 (6) (1905), 139-216.

[23] Open problems in topology II /ed. by Elliott Pearl/ Elsevier, 2007.

[24] C. A. Rogers, Functions of the first Baire class, J. London Math. Soc. 37 (3) (1988), $535-544$.

[25] S. Rolewicz, On inversion of non-linear transformations, Studia Math. 17 (1958), 79-83.

[26] H.R. Shatery, J. Zafarani, The equality between Borel and Baire classes, Real Anal. Exch. 30 (1) (2004-2005), 373-384.

[27] C. Stegall, Functins of the first Baire class with values in Banach spaces, Proc. Amer. Math. Soc. 111 (1991), 981-991.

[28] A.H. Stone, Paracompactness and product spaces, Bull. Amer. Math. Soc. 54 (10) (1948), 977-982.

[29] L. Veselý, Characterization of Baire-one functions between topological spaces, Acta Univ. Carol., Math. Phys. 33 (2) (1992), 143-156. 
Department of Mathematical Analysis, Faculty of Mathematics and Informatics, Chernivtsi National University, Chernivtsi, Ukraine

E-mail address: maslenizza.ua@gmail.com 